\title{
Identification of the inertia matrix of a rotating body based on errors-in-variables models
}

\author{
Byung-Eul Jun ${ }^{1, *,}$, Dennis S. Bernstein ${ }^{2}$ and N. Harris McClamroch ${ }^{2}$ \\ ${ }^{1}$ Guidance and Control Directorate, Agency for Defense Development, Daejeon 305-600, Korea \\ ${ }^{2}$ Department of Aerospace Engineering, University of Michigan, Ann Arbor, MI 48109, U.S.A.
}

\begin{abstract}
SUMMARY
This paper proposes a procedure for identifying the inertia matrix of a rotating body. The procedure based on Euler's equation governing rotational motion assumes errors-in-variables models in which all measurements, torque as well as angular velocities, are corrupted by noises. In order for consistent estimation, we introduce an extended linear regression model by augmenting the regressors with constants and the parameters with noise-contributed terms. A transformation, based on low-pass filtering, of the extended model cancels out angular acceleration terms in the regressors. Applying the method of least correlation to the model identifies the elements of the inertia matrix. Analysis shows that the estimates converge to the true parameters as the number of samples increases to infinity. Monte Carlo simulations demonstrate the performance of the algorithm and support the analytical consistency. Copyright (C) 2009 John Wiley \& Sons, Ltd.
\end{abstract}

Received 4 June 2008; Revised 3 November 2008; Accepted 23 December 2008

KEY WORDS: identification; inertia matrix; errors in variables; least-correlation estimate; nonlinear identification

\section{INTRODUCTION}

It is a trend to use smaller, lighter and cheaper instruments for systems, which usually means that measurements are more corrupted by noise. This is true for uninhabited air or space vehicles, where there is a premium on size and weight. Control requirements, however, may be stricter than those of conventional vehicles in order to meet the needs for clustering or formation flight $[1,2]$. Many existing algorithms

\footnotetext{
*Correspondence to: Byung-Eul Jun, Guidance and Control Directorate, Agency for Defense Development, Daejeon 305-600, Korea.

†E-mail: bejun@add.re.kr, beuljun@gmail.com

Contract/grant sponsor: The National Science Foundation; contract/grant number: F006769
}

for identifying inertial parameters in space use the method of least squares [3-7], however, this technique exhibits high sensitivity to errors in regressors [8]. The regressors in identification models are composed of measurements, such as angular velocity, angular acceleration and attitude, which are not free from noise [3-7]. The models, where input as well as output measurements are contaminated by noise, are known as errors-in-variables (EIV) models $[9,10]$. It is known that the least-squares method tends to generate error-prone estimates for EIV models [8]. Making the problem worse is that the regressors in the estimation models are not linear in the measurements when the models are based on Euler equation $[3,4,7]$ or derived from angular-momentum conservation $[5,6]$. The method of least correlation [11] has a capability to cope with the noisy measurements of all variables provided that the regressors are linear in 
the variables. An extension of this method provides consistent estimates for nonlinear systems described by polynomials in the variables [12].

This paper describes an application of the leastcorrelation methods for identifying the inertia matrix of a rotating body. In this work we assume that the external torque and angular velocity are measured with noise, but the angular acceleration is not available. The estimation model based on Euler's equation is formulated via two steps, specifically, by extending the linear regression model by augmenting the regressors with constants and the parameters with noise-contributed terms, and transforming the extended model to an equivalent form without angular acceleration terms. Applying the method of least correlation to the extended and transformed model provides an algorithm identifying the inertia matrix of a rotating body with consistency.

Analysis shows that the procedure gives consistent estimates, that is, the estimates converge to the true parameters as the number of samples increases to infinity. Simulation results, for example, confirm the performance of the estimation method numerically. The estimation method introduced in this work can be applied to various kinds of systems such as spacecraft [5-7, 13], robots [3] and other rotating structures $[4,14]$.

The content is organized as follows. Section 2 provides the model, problem statement and assumptions. Section 3 describes the main results including the estimation procedure for the inertia matrix and a performance analysis of the estimation algorithm. The results of Monte Carlo simulation are reported in Section 4. Concluding remarks are stated in Section 5.

\section{PROBLEM AND ASSUMPTIONS}

The rotational motion of a rigid body is governed by Euler's equation

$$
J \dot{\omega}_{*}(t)+\omega_{*}(t) \times J \omega_{*}(t)=M_{*}(t)
$$

where $J \in \mathbb{R}^{3 \times 3}$ denotes the inertia matrix which is constant, symmetric and positive definite, $\omega_{*}(t) \in \mathbb{R}^{3}$ is the angular velocity vector and $M_{*}(t) \in \mathbb{R}^{3}$ is the external moment (or torque) acting on the body about

Copyright (C) 2009 John Wiley \& Sons, Ltd. its mass center. Let $\omega(t) \in \mathbb{R}^{3}$ and $M(t) \in \mathbb{R}^{3}$ denote the measurements of $\omega_{*}(t)$ and $M_{*}(t)$, respectively, that is,

$$
\begin{gathered}
\omega(t) \triangleq \omega_{*}(t)+\zeta(t) \\
M(t) \triangleq M_{*}(t)+\eta(t)
\end{gathered}
$$

where $\zeta(t) \in \mathbb{R}^{3}$ and $\eta(t) \in \mathbb{R}^{3}$ are measurement noises. We assume that $\omega(t)$ is measured, but $\dot{\omega}(t)$ is not. Our goal is to identify all components of $J$ by using $\omega(t)$ and $M(t)$.

Measurements are frequently described as stochastic processes with deterministic components. To provide a common framework for deterministic and stochastic signals [15, pp. 33-34], we assume that all measurements are quasi-stationary and employ the notation

$$
\bar{E}[f(k h)] \triangleq \lim _{N \rightarrow \infty} \frac{1}{N} \sum_{k=1}^{N} E[f(k h)]
$$

for discrete-time signal $f(k h), k=1,2, \ldots, N$ with sampling interval $h$, where $E$ denotes expectation. We implicitly assume that the limit in (4) exists.

We introduce the following assumptions.

A1. The measurements $\omega(k h)$ and $M(k h)$ are quasi-stationary and jointly quasi-stationary $[15$, p. 34].

A2. The noises $\zeta(k h)$ and $\eta(k h)$ are zero-mean and finitely cross-correlated with $\omega(k h)$, that is, there exists the smallest $\tau>0$ such that

$$
\begin{array}{ll}
\bar{E}\left[\omega(k h) \zeta^{\mathrm{T}}(k h-s h)\right]=0 & \text { for all }|s|>\tau \\
\bar{E}\left[\omega(k h) \eta^{\mathrm{T}}(k h-s h)\right]=0 & \text { for all }|s|>\tau
\end{array}
$$

A3. For $\tau$ given by A2, $\omega(k h)$ satisfies

$$
\operatorname{rank}\left\{\bar{R}_{\omega \omega}\left(k, k^{\prime}, N\right)+\bar{R}_{\omega \omega}\left(k^{\prime}, k, N\right)\right\}=3
$$

where $k^{\prime}=k-\tau, N$ denotes the number of samples and the empirical correlation $\bar{R}_{\omega \omega}\left(k_{1}, k_{2}, N\right)$ is defined by

$$
\bar{R}_{\omega \omega}\left(k_{1}, k_{2}, N\right) \triangleq \frac{1}{N_{\tau}} \sum_{k=1+\tau}^{N} \omega\left(k_{1} h\right) \omega^{\mathrm{T}}\left(k_{2} h\right)
$$

where $N_{\tau}=N-\tau$ and $k_{1}=k, k_{2}=k-\tau$ or $k_{1}=$ $k-\tau, k_{2}=k$.

Int. J. Adapt. Control Signal Process. 2010; 24:203-210 


\section{ESTIMATION OF THE INERTIA MATRIX}

By using (2) and (3), (1) is written as

$$
J(\dot{\omega}-\dot{\zeta})+(\omega-\zeta) \times J(\omega-\zeta)=M-\eta
$$

where the time argument $t$ is omitted for convenience. For an arbitrary vector $x \triangleq\left[\begin{array}{lll}x_{1} & x_{2} & x_{3}\end{array}\right]^{\mathrm{T}}$, let us define two operators [16]

$$
\begin{gathered}
L(x) \triangleq\left[\begin{array}{cccccc}
x_{1} & 0 & 0 & 0 & x_{3} & x_{2} \\
0 & x_{2} & 0 & x_{3} & 0 & x_{1} \\
0 & 0 & x_{3} & x_{2} & x_{1} & 0
\end{array}\right] \\
x \triangleq \triangleq\left[\begin{array}{ccc}
0 & -x_{3} & x_{2} \\
x_{3} & 0 & -x_{1} \\
-x_{2} & x_{1} & 0
\end{array}\right]
\end{gathered}
$$

and a parameter vector

$$
\theta \triangleq\left[\begin{array}{llllll}
J_{11} & J_{22} & J_{33} & J_{23} & J_{13} & J_{12}
\end{array}\right]^{\mathrm{T}}
$$

so that $J x=L(x) \theta$. Then with the regressor matrix $\phi(t) \in \mathbb{R}^{6 \times 3}$ defined by

$$
\phi^{\mathrm{T}}(t) \triangleq L(\dot{\omega}-\dot{\zeta})+(\omega-\zeta)^{\times} L(\omega-\zeta)
$$

Equation (9) is equivalent to the linear regression equation

$$
z(t)=\phi^{\mathrm{T}}(t) \theta
$$

where $z(t) \triangleq M_{*}(t)=M(t)-\eta(t)$.

Let us split $\phi(t)$ into three parts as

$$
\phi(t)=\psi(t)-\delta(t)-\xi(t)
$$

where

$$
\begin{gathered}
\psi^{\mathrm{T}}(t) \triangleq L(\dot{\omega})+\omega^{\times} L(\omega) \\
\delta^{\mathrm{T}}(t) \triangleq L(\dot{\zeta})+\zeta^{\times} L(\zeta) \\
\xi^{\mathrm{T}}(t) \triangleq \omega_{*}^{\times} L(\zeta)+\zeta^{\times} L\left(\omega_{*}\right)
\end{gathered}
$$

\section{Letting}

$$
\begin{aligned}
& \omega_{*}(t) \triangleq\left[\begin{array}{lll}
p_{*} & q_{*} & r_{*}
\end{array}\right]^{\mathrm{T}}, \quad \omega(t) \triangleq\left[\begin{array}{lll}
p & q & r
\end{array}\right]^{\mathrm{T}} \\
& \zeta(t) \triangleq\left[\begin{array}{lll}
\zeta_{p} & \zeta_{q} & \zeta_{r}
\end{array}\right]^{\mathrm{T}}
\end{aligned}
$$

gives

$$
\begin{array}{r}
\psi^{\mathrm{T}} \triangleq\left[\begin{array}{cccccc}
\dot{p} & -q r & q r & q^{2}-r^{2} & \dot{r}+p q & \dot{q}-r p \\
r p & \dot{q} & -r p & \dot{r}-p q & r^{2}-p^{2} & \dot{p}+q r \\
-p q & p q & \dot{r} & \dot{q}+r p & \dot{p}-q r & p^{2}-q^{2}
\end{array}\right] \\
\xi^{\mathrm{T} \triangleq} \triangleq\left[\begin{array}{cccccc}
\dot{\zeta}_{p} & -\zeta_{q} \zeta_{r} & \zeta_{q} \zeta_{r} & \zeta_{q}^{2}-\zeta_{r}^{2} & \dot{\zeta}_{r}+\zeta_{p} \zeta_{q} & \dot{\zeta}_{q}-\zeta_{r} \zeta_{p} \\
\zeta_{r} \zeta_{p} & \dot{\zeta}_{q} & -\zeta_{r} \zeta_{p} & \dot{\zeta}_{r}-\zeta_{p} \zeta_{q} & \zeta_{r}^{2}-\zeta_{p}^{2} & \dot{\zeta}_{p}+\zeta_{q} \zeta_{r} \\
-\zeta_{p} \zeta_{q} & \zeta_{p} \zeta_{q} & \dot{\zeta}_{r} & \dot{\zeta}_{q}+\zeta_{r} \zeta_{p} & \dot{\zeta}_{p}-\zeta_{q} \zeta_{r} & \zeta_{p}^{2}-\zeta_{q}^{2}
\end{array}\right] \\
+\left[\begin{array}{ccccccc}
0 & -\zeta_{q} r_{*} & \zeta_{q} r_{*} & \zeta_{q} q_{*}-\zeta_{r} r_{*} & \zeta_{p} q_{*} & -\zeta_{r} p_{*} \\
\zeta_{r} p_{*} & 0 & -\zeta_{r} p_{*} & -\zeta_{p} q_{*} & \zeta_{r} r_{*}-\zeta_{p} p_{*} & \zeta_{q} r_{*} \\
-\zeta_{p} q_{*} & \zeta_{p} q_{*} & 0 & \zeta_{r} p_{*} & -\zeta_{q} r_{*} & \zeta_{p} p_{*}-\zeta_{q} q_{*}
\end{array}\right] \\
+\left[\begin{array}{ccccccc}
0 & -q_{*} \zeta_{r} & q_{*} \zeta_{r} & q_{*} \zeta_{q}-r_{*} \zeta_{r} & p_{*} \zeta_{q} & -r_{*} \zeta_{p} \\
r_{*} \zeta_{p} & 0 & -r_{*} \zeta_{p} & -p_{*} \zeta_{q} & r_{*} \zeta_{r}-p_{*} \zeta_{p} & q_{*} \zeta_{r} \\
-p_{*} \zeta_{q} & p_{*} \zeta_{q} & 0 & r_{*} \zeta_{p} & -q_{*} \zeta_{r} & p_{*} \zeta_{p}-q_{*} \zeta_{q}
\end{array}\right]
\end{array}
$$

Int. J. Adapt. Control Signal Process. 2010; 24:203-210 DOI: $10.1002 /$ acs 
Substituting (15) into (14) yields an EIV model

$$
\begin{gathered}
y(t)=\psi^{\mathrm{T}}(t) \theta+e(t) \\
e(t)=\eta(t)-\delta^{\mathrm{T}}(t) \theta-\xi^{\mathrm{T}}(t) \theta
\end{gathered}
$$

where $y(t) \triangleq M(t)$.

Let us consider how to treat the noise included in $\psi(t)$. Assume for the moment that $\dot{\omega}(t)$ is given. Employing the method of least correlation for (22) and (23) yields estimates with biases even if the noises satisfy A2. The biases come from $\zeta(t)$ since the second term on the right-hand side of (17) contains quadratic components of $\zeta_{p}, \zeta_{q}, \zeta_{r}[11,12]$. Let us consider an augmented regressor matrix $\psi_{a}(t) \in \mathbb{R}^{9 \times 3}$ and an extended parameter vector $\theta_{a}(t) \in \mathbb{R}^{9}$ defined by

$$
\begin{gathered}
\psi_{a}(t)=\left[\begin{array}{lll}
\psi^{\mathrm{T}}(t) & -I_{3}
\end{array}\right]^{\mathrm{T}} \\
\theta_{a}(t)=\left[\begin{array}{llll}
\theta^{\mathrm{T}} & \theta_{7}(t) & \theta_{8}(t) & \theta_{9}(t)
\end{array}\right]^{\mathrm{T}}
\end{gathered}
$$

respectively, where $I_{3} \in \mathbb{R}^{3 \times 3}$ is the identity matrix and $\theta_{7}, \theta_{8}, \theta_{9}$ are given by

$$
\begin{aligned}
\theta_{7} \triangleq & -J_{22} \zeta_{q} \zeta_{r}+J_{33} \zeta_{q} \zeta_{r}+J_{23}\left(\zeta_{q}^{2}-\zeta_{r}^{2}\right) \\
& +J_{13} \zeta_{p} \zeta_{q}-J_{12} \zeta_{r} \zeta_{p} \\
\theta_{8} \triangleq & J_{11} \zeta_{r} \zeta_{p}-J_{33} \zeta_{r} \zeta_{p}-J_{23} \zeta_{p} \zeta_{q} \\
& +J_{13}\left(\zeta_{r}^{2}-\zeta_{p}^{2}\right)+J_{12} \zeta_{q} \zeta_{r} \\
\theta_{9} \triangleq & -J_{11} \zeta_{p} \zeta_{q}+J_{22} \zeta_{p} \zeta_{q}+J_{23} \zeta_{r} \zeta_{p} \\
& -J_{13} \zeta_{q} \zeta_{r}+J_{12}\left(\zeta_{p}^{2}-\zeta_{q}^{2}\right)
\end{aligned}
$$

Using (24)-(25) gives the extended EIV model

$$
\begin{gathered}
y(t)=\psi_{a}^{\mathrm{T}}(t) \theta_{a}(t)+e_{a}(t) \\
e_{a}(t)=\eta(t)-L(\dot{\zeta}) \theta-\xi^{\mathrm{T}}(t) \theta
\end{gathered}
$$

Note that $\psi_{a}(t)$ in (29) is not available because $\dot{\omega}(t)$ is not measured by assumption. With the differential operator $p \triangleq \mathrm{d} / \mathrm{d} t$ and a constant $\gamma>0$, we introduce the low-pass filter [17, p. 284]

$$
\lambda=\frac{1}{1+\gamma p}
$$

Copyright (C) 2009 John Wiley \& Sons, Ltd. in order to remove $\dot{\omega}(t)$ from (29)-(30). Applying the operator (31) to (29)-(30) and introducing

$$
\begin{gathered}
\psi_{a_{f}}(t) \triangleq\left[\begin{array}{ll}
\psi_{f}^{\mathrm{T}}(t) & -I_{3}
\end{array}\right]^{\mathrm{T}} \\
\theta_{a_{f}}(t) \triangleq\left[\begin{array}{ll}
\theta^{\mathrm{T}} & \theta_{[7-9]_{f}}^{\mathrm{T}}(t)
\end{array}\right]^{\mathrm{T}}
\end{gathered}
$$

with

$$
\begin{gathered}
\psi_{f}^{\mathrm{T}}(t) \triangleq L\left(\frac{\omega-\omega_{f}}{\gamma}\right)+\lambda\left(\omega^{\times} L(\omega)\right) \\
\theta_{[7-9]_{f}}(t) \triangleq\left[\theta_{7_{f}}(t) \quad \theta_{8_{f}}(t) \quad \theta_{9_{f}}(t)\right]^{\mathrm{T}}
\end{gathered}
$$

yields the EIV regression model

$$
\begin{gathered}
y_{f}(t)=\psi_{a_{f}}^{\mathrm{T}}(t) \theta_{a_{f}}(t)+e_{a_{f}}(t) \\
e_{a_{f}}(t)=\eta_{f}(t)-L\left(\frac{\zeta-\zeta_{f}}{\gamma}\right) \theta-\xi_{f}^{\mathrm{T}}(t) \theta
\end{gathered}
$$

since $\left[\lambda\left(\psi_{a}^{\mathrm{T}}(t) \theta_{a}(t)\right)\right](t)=\psi_{a_{f}}^{\mathrm{T}}(t) \theta_{a_{f}}(t)$, where $(\cdot)_{f}(t) \triangleq$ $[\lambda(\cdot)](t),(\cdot)_{f}(0) \triangleq 0$. Note that $\psi_{a_{f}}(t)$ and $e_{a_{f}}(t)$ do not contain $\dot{\omega}(t)$ and $\dot{\zeta}$, respectively.

We now work with sampled measurements. Given an arbitrary estimate $\bar{\theta}_{a_{f}}$, consider the criterion

$$
J^{2}=\left(\frac{1}{N_{\tau}}\left(Y_{0}-\Psi_{0} \bar{\theta}_{a_{f}}\right)^{\mathrm{T}}\left(Y_{\tau}-\Psi_{\tau} \bar{\theta}_{a_{f}}\right)\right)^{2}
$$

where $Y_{0}, Y_{\tau} \in \mathbb{R}^{3 N_{\tau}}$ and $\Psi_{0}, \Psi_{\tau} \in \mathbb{R}^{3 N_{\tau} \times 9}$ are defined by

$$
\begin{gathered}
Y_{0} \triangleq\left[\begin{array}{c}
y_{f}(N h) \\
y_{f}\left(N_{1} h\right) \\
\vdots \\
y_{f}(h+\tau h)
\end{array}\right], \quad Y_{\tau} \triangleq\left[\begin{array}{c}
y_{f}\left(N_{\tau} h\right) \\
y_{f}\left(N_{\tau+1} h\right) \\
\vdots \\
y_{f}(h)
\end{array}\right] \\
\Psi_{0} \triangleq\left[\begin{array}{c}
\psi_{a_{f}}^{\mathrm{T}}(N h) \\
\psi_{a_{f}}^{\mathrm{T}}\left(N_{1} h\right) \\
\vdots \\
\psi_{a_{f}}^{\mathrm{T}}(h+\tau h)
\end{array}\right], \quad \Psi_{\tau} \triangleq\left[\begin{array}{c}
\psi_{a_{f}}^{\mathrm{T}}\left(N_{\tau} h\right) \\
\psi_{a_{f}}^{\mathrm{T}}\left(N_{\tau+1} h\right) \\
\vdots \\
\psi_{a_{f}}^{\mathrm{T}}(h)
\end{array}\right]
\end{gathered}
$$


Note that $J$ is an empirical correlation between the residuals of the estimate $\bar{\theta}_{a_{f}}$. When $\tau=0, J$ yields the least-squares estimate.

Minimizing (38) with respect to $\bar{\theta}_{a_{f}}$ gives

$$
\hat{\theta}_{a_{f}}(\tau, N)=\left(\Psi_{0 / \tau}^{\mathrm{T}} \Psi_{\tau / 0}\right)^{-1} \Psi_{0 / \tau}^{\mathrm{T}} Y_{\tau / 0}
$$

where

$$
\Psi_{0 / \tau} \triangleq\left[\begin{array}{c}
\Psi_{0} \\
\Psi_{\tau}
\end{array}\right], \quad \Psi_{\tau / 0} \triangleq\left[\begin{array}{c}
\Psi_{\tau} \\
\Psi_{0}
\end{array}\right], \quad Y_{\tau / 0} \triangleq\left[\begin{array}{c}
Y_{\tau} \\
Y_{0}
\end{array}\right]
$$

The matrix $\Psi_{0 / \tau}^{\mathrm{T}} \Psi_{\tau / 0} \in \mathbb{R}^{9 \times 9}$ in (41), given as

$$
\begin{aligned}
& \Psi_{0 / \tau}^{\mathrm{T}} \Psi_{\tau / 0} \\
& =\sum_{k=1+\tau}^{N}\left[\begin{array}{cc}
\psi_{f}(k h) \psi_{f}^{\mathrm{T}}((k-\tau) h) & -\psi_{f}(k h) \\
-\psi_{f}^{\mathrm{T}}((k-\tau) h) & I_{3}
\end{array}\right] \\
& \quad+\sum_{k=1+\tau}^{N}\left[\begin{array}{cc}
\psi_{f}((k-\tau) h) \psi_{f}^{\mathrm{T}}(k h) & -\psi_{f}((k-\tau) h) \\
-\psi_{f}^{\mathrm{T}}(k h) & I_{3}
\end{array}\right]
\end{aligned}
$$

is nonsingular due to A3. The estimate (41) has the following property.

\section{Theorem 1}

Suppose that $\mathrm{A} 1-\mathrm{A} 3$ are satisfied. Then, for all $k$, as $N$ goes to infinity, the least-correlation estimate (41) for the model (36)-(37) converges to the expectation of $\theta_{a_{f}}(k h)$, that is,

$$
\lim _{N \rightarrow \infty} \hat{\theta}_{a_{f}}(\tau, N)=E\left[\theta_{a_{f}}(k h)\right]
$$

\section{Proof}

The proof is sketched in Appendix A.

Note that (41) is a consistent estimate of $\theta$, which is clear from the componentwise expression of (43) written as

$$
\lim _{N \rightarrow \infty}\left[\begin{array}{c}
\hat{\theta}(\tau, N) \\
\hat{\theta}_{[7-9]_{f}}(\tau, N)
\end{array}\right]=\left[\begin{array}{c}
\theta \\
E\left[\theta_{[7-9]_{f}}(k h)\right]
\end{array}\right]
$$

If the components of $\zeta(k h)$ are independent, identically distributed (i.i.d.) with the same variance, then augmenting the regressor matrix and extending the parameter vector are not necessary since $\bar{E}\left[\theta_{i}(k h)\right]=$ $0, i=7,8,9$ for all $k$ from (26)-(28). That is, the estimate

$$
\hat{\theta}_{f}(\tau, N)=\left(\Psi_{0 / \tau}^{\mathrm{oT}} \Psi_{\tau / 0}^{\mathrm{o}}\right)^{-1} \Psi_{0 / \tau}^{\mathrm{oT}} Y_{\tau / 0}
$$

is consistent, where the relevant matrices are defined by

$$
\begin{gathered}
\Psi_{0 / \tau}^{\mathrm{o}} \triangleq\left[\begin{array}{c}
\Psi_{0}^{\mathrm{o}} \\
\Psi_{\tau}^{\mathrm{o}}
\end{array}\right], \quad \Psi_{\tau / 0}^{\mathrm{o}} \triangleq\left[\begin{array}{c}
\Psi_{\tau}^{\mathrm{o}} \\
\Psi_{0}^{\mathrm{o}}
\end{array}\right] \\
\Psi_{0}^{\mathrm{o}} \triangleq\left[\begin{array}{c}
\psi_{f}^{\mathrm{T}}(N h) \\
\psi_{f}^{\mathrm{T}}\left(N_{1} h\right) \\
\vdots \\
\psi_{f}^{\mathrm{T}}(h+\tau h)
\end{array}\right], \quad \Psi_{\tau}^{\mathrm{o} \triangleq}\left[\begin{array}{c}
\psi_{f}^{\mathrm{T}}\left(N_{\tau} h\right) \\
\psi_{f}^{\mathrm{T}}\left(N_{\tau+1} h\right) \\
\vdots \\
\psi_{f}^{\mathrm{T}}(h)
\end{array}\right]
\end{gathered}
$$

\section{NUMERICAL EXAMPLES}

Consider a body with the inertia matrix

$$
J=\left[\begin{array}{ccc}
1.20 & 0.11 & -0.18 \\
0.11 & 1.70 & 0.16 \\
-0.18 & 0.16 & 2.13
\end{array}\right]
$$

The system (1) with (48) is driven by $M_{*}(t)$ in Figure 1. We assume that $\eta(t)=0$ since it does not contribute to the bias errors of identification. In each simulation, we sample $M(t)$ and $\omega(t)$ at every $10 \mathrm{~ms}$. The measurement noise $\zeta(t)$ is Gaussian with zero mean and covariance

$$
\operatorname{Cov}\left[\zeta(t) \zeta^{\mathrm{T}}(t)\right]=\left[\begin{array}{ccc}
\sigma_{p}^{2} & 0 & 0 \\
0 & \sigma_{q}^{2} & 0 \\
0 & 0 & \sigma_{r}^{2}
\end{array}\right]
$$

For this example, we consider two cases of measurement noises which are finitely and infinitely correlated. In the first case the input measurements are corrupted as

$$
\omega(t) \triangleq \omega_{*}(t)+\beta \zeta(t)+(1-\beta) \zeta(t-1)
$$

with $\beta=0.7$. As $\omega(k h)$ is not correlated with $\omega((k-$ $\tau) h$ ) when $|\tau| \geqslant 2$, we choose $\tau=2$ or $\tau h=0.02 \mathrm{~s}$. The

Int. J. Adapt. Control Signal Process. 2010; 24:203-210 DOI: $10.1002 / \mathrm{acs}$ 


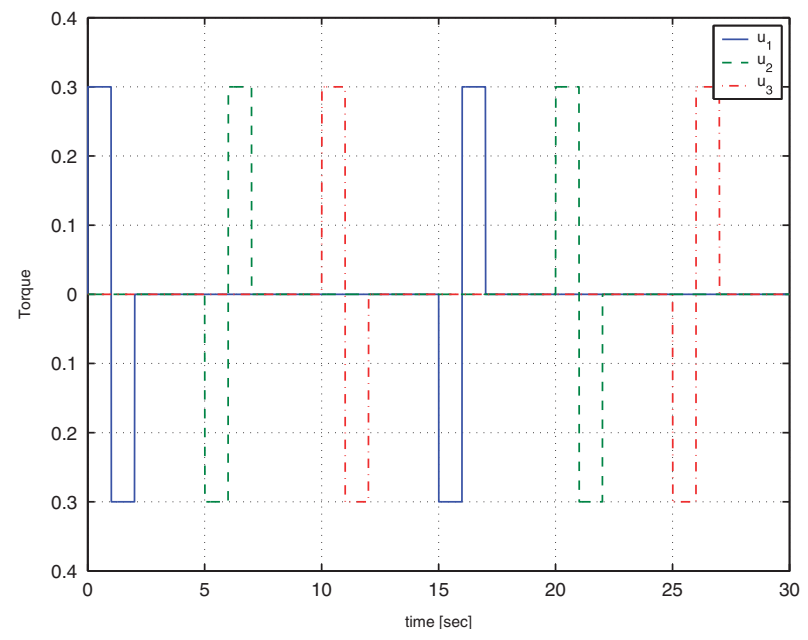

Figure 1 . Moment $M(t)$ applied to the body.

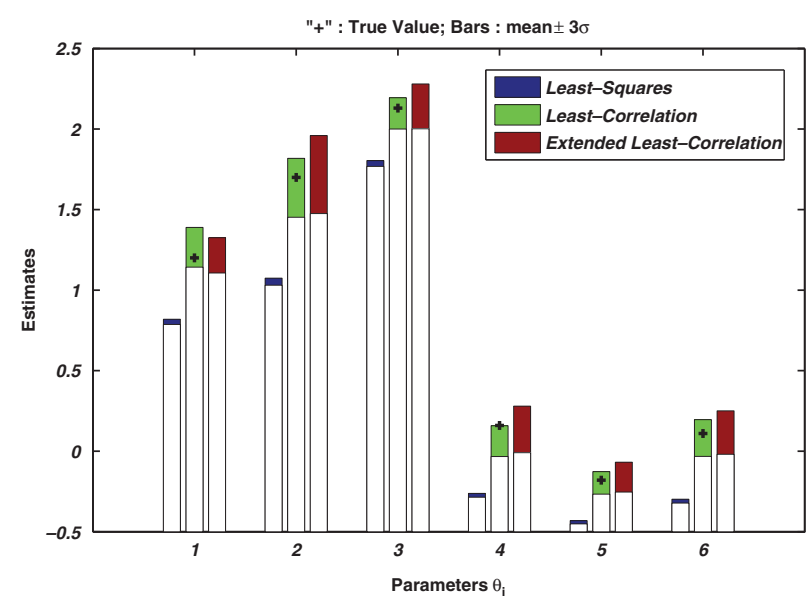

Figure 2. Identification results from measurements corrupted by the finitely correlated noise (49) with $\left(\sigma_{p}, \sigma_{q}, \sigma_{r}\right)=(0.4,0.3,0.2) \mathrm{deg} / \mathrm{s}$.

value $\gamma=10$ is chosen to provide a small bandwidth for the low-pass filter (31). In order to identify the inertia matrix (48) we employ three algorithms, the least-correlation estimate (45), the least-squares estimate, which is obtained from (45) by setting $\tau=0$, and the extended least-correlation estimate in (41).

Figure 2 summarizes simulation results from the algorithms. Each bar in Figure 2 represents the possible

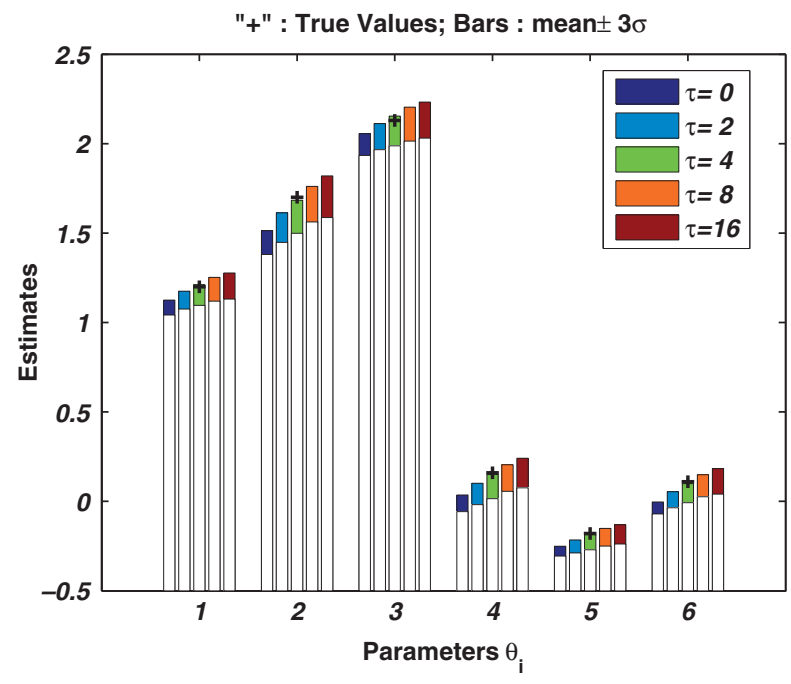

Figure 3. Identification results from measurements corrupted by the infinitely correlated noise (50) with $\left(\sigma_{p}, \sigma_{q}, \sigma_{r}\right)=(0.4,0.3,0.2) \mathrm{deg} / \mathrm{s}$.

range of each parameter identified by the corresponding algorithm. The maximum and minimum values of each bar are evaluated by $\bar{E}\left[\hat{\theta}_{i}\right] \pm \bar{\sigma}\left(\hat{\theta}_{i}\right)$ from 100 empirical Monte Carlo runs for each, where $\bar{E}$ and $\bar{\sigma}$ denote the empirical mean and the standard deviation, respectively. Figure 2 confirms that the extended least-correlation estimates, (41) and (45), outperform the conventional least squares as well as the standard least-correlation estimate. All $3 \sigma$ ranges of the least-squares estimates in Figure 2 are out of their true parameters. Both the standard and extended least-correlation algorithms generate good estimates, but the extended version tends to give estimates with less bias than the standard.

According to Theorem 1, (41) gives consistent estimates provided that the measurement noise in regressor matrix is at most finitely correlated. We, however, try to show numerically that the estimates can be applied to problems with infinitely correlated noise which is violating A2. For this case we use the angular velocity measurements given by

$$
\omega(t) \triangleq \omega_{*}(t)+\frac{1}{1+\beta p} \zeta(t)
$$


instead of (2), where $\beta=0.04 \mathrm{~s}$. Figure 3 shows that the extended least-correlation algorithm works well on the infinitely correlated noise provided that A3 is satisfied with a large value of $\tau$. According to the estimates in Figure 3 with $\tau h=0.16 \mathrm{~s}(\tau=16)$, which is four times of $\beta=0.04$, the algorithm gives almost bias-free results. This result implies that the extended least-correlation algorithm can be useful for measurements which are corrupted by infinitely correlated noises.

\section{CONCLUDING REMARKS}

This paper introduces a procedure for identifying the inertia matrix of a rotating body. The estimation algorithm is based on the Euler equation governing rotational motion and assumes errors-in-variables (EIV) models in which all variables are corrupted by noise. The main idea is composed of three steps-extending regressors and parameters, filtering out angular acceleration terms and employing the method of least correlation. In the first step, the regressor matrix is augmented by the identity matrix with proper size and the parameter vector is augmented by terms contributed by noisy measurements. The second step is to transform the EIV model of the Euler equation, which contains angular acceleration terms, to a model that does not contain the components. The last step, employing the method of least correlation to the extended and transformed model, gives an estimate of the inertia matrix. Analysis shows that the estimates are consistent in the sense that the estimates converge to the true values as the number of samples increases to infinity. Monte Carlo simulations demonstrate the performance and support the analytical results.

Given the measurements of translational accelerations, attitude angles and external forces of a translating and rotating rigid body, the proposed algorithm can be extended to the problem [3] which identifies all inertial parameters including mass and center of mass as well as inertia matrix. We expect that this extension gives reasonable results even though the estimates are not free from bias. If the attitude angles are measured almost free from noise, then the proposed procedure gives good estimates of the complete set of inertial parameters.

Copyright (C) 2009 John Wiley \& Sons, Ltd.

\section{APPENDIX A}

We sketch the proof of Theorem 1. Equation (41) is equivalent to

$$
\begin{aligned}
\hat{\theta}_{a_{f}}(\tau, N)= & \left(\bar{R}_{\psi_{a_{f}} \psi_{a_{f}}}\left(k_{1}, k_{2}\right)+\bar{R}_{\psi_{a_{f}} \psi_{a_{f}}}\left(k_{2}, k_{1}\right)\right)^{-1} \\
& \times\left(\bar{r}_{\psi_{a_{f}} y_{f}}\left(k_{1}, k_{2}\right)+\bar{r}_{\psi_{a_{f}} y_{f}}\left(k_{2}, k_{1}\right)\right)
\end{aligned}
$$

where the empirical correlations are defined by

$$
\begin{gathered}
\bar{R}_{\psi_{a_{f}} \psi_{a_{f}}}\left(k_{1}, k_{2}\right) \triangleq \frac{1}{N_{\tau}} \sum_{k=1+\tau}^{N} \psi_{a_{f}}\left(k_{1} h\right) \psi_{a_{f}}^{\mathrm{T}}\left(k_{2} h\right) \\
\bar{r}_{\psi_{a_{f}} y_{f}}\left(k_{1}, k_{2}\right) \triangleq \frac{1}{N_{\tau}} \sum_{k=1+\tau}^{N} \psi_{a_{f}}\left(k_{1} h\right) y_{f}\left(k_{2} h\right)
\end{gathered}
$$

with either $k_{1}=k, k_{2}=k-\tau$ or $k_{1}=k-\tau, k_{2}=k$. Using the discrete-time equivalence of (36) to $\bar{r}_{\psi_{a_{f}} y_{f}}\left(k_{1}, k_{2}\right)$ gives

$$
\begin{aligned}
\bar{r}_{\psi_{a_{f}} y_{f}}\left(k_{1}, k_{2}\right)= & \overline{\mathbf{t}}_{\psi_{a_{f}} \psi_{a_{f}} \theta_{a_{f}}}\left(k_{1}, k_{2}, k_{2}\right) \\
& +\bar{r}_{\psi_{a} e_{a_{f}}}\left(k_{1}, k_{2}\right)
\end{aligned}
$$

where the empirical bicorrelation $\overline{\mathbf{t}}_{\psi_{a_{f}} \psi_{a_{f}} \theta_{a_{f}}}$ [18] and $\bar{r}_{\psi_{a_{f}} e_{a_{f}}}$ are defined by

$$
\begin{aligned}
& \overline{\mathbf{t}}_{\psi_{a_{f}} \psi_{a_{f}} \theta_{a_{f}}} \triangleq \frac{1}{N_{\tau}} \sum_{k=1+\tau}^{N} \psi_{a_{f}}\left(k_{1} h\right) \psi_{a_{f}}^{\mathrm{T}}\left(k_{2} h\right) \theta_{a_{f}}\left(k_{2} h\right) \\
& \bar{r}_{\psi_{a_{f}} e_{a_{f}}} \triangleq \frac{1}{N_{\tau}} \sum_{k=1+\tau}^{N} \psi_{a_{f}}\left(k_{1} h\right) e_{a_{f}}\left(k_{2} h\right)
\end{aligned}
$$

respectively.

When $N$ goes to infinity, $\bar{R}_{\psi_{a_{f}} \psi_{a_{f}}}\left(k_{1}, k_{2}\right)$ converges to $R_{\psi_{a_{f}} \psi_{a_{f}}}(\tau)$ due to the ergodic theory [15, Theorem 2.3 in p. 43] and A1, i.e.

$$
\lim _{N \rightarrow \infty} \bar{R}_{\psi_{a_{f}} \psi_{a_{f}}}\left(k_{1}, k_{2}\right)=R_{\psi_{a_{f}} \psi_{a_{f}}}(\tau)
$$

Applying $N \rightarrow \infty$ to (A2) yields

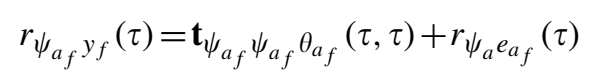


where each term is evaluated as follows:

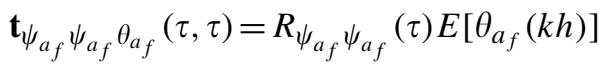

$$
\begin{aligned}
& r_{\psi_{a_{f}} e_{a_{f}}}(\tau)=0
\end{aligned}
$$

Using (A4)-(A6), $\quad \bar{r}_{\psi_{a_{f}} y_{f}}\left(k_{1}, k_{2}\right) \quad$ at $\quad N \rightarrow \infty$ is expressed as

$$
\lim _{N \rightarrow \infty} \bar{r}_{\psi_{a_{f}} y_{f}}\left(k_{1}, k_{2}\right)=R_{\psi_{a_{f}} \psi_{a_{f}}}(\tau) E\left[\theta_{a_{f}}(k h)\right](\mathrm{A} 7)
$$

for all $k$. Applying (A3) and (A7) to

$$
\lim _{N \rightarrow \infty} \hat{\theta}_{a_{f}}(\tau, N)=R_{\psi_{a_{f}} \psi_{a_{f}}}^{-1}(\tau) r_{\psi_{a_{f}} y_{f}}(\tau)
$$

which is an expression of (A1) at $N \rightarrow \infty$, yields (43).

\section{REFERENCES}

1. Giulietti F, Pollini L, Innocenti M. Autonomous formation flight. IEEE Control Systems Magazine 2000; 20(6): 34-44.

2. Zetocha P, Self L, Wainwright R, Burns R. Commanding and controlling satellite clusters. IEEE Intelligent Systems 2000; 15(6):8-13.

3. Hahn H, Niebergall M. Development of a measurement robot for identifying all inertia parameters of a rigid body in a single experiment. IEEE Transactions on Control Systems Technology 2001; 9(2):416-423.

4. Kim B, Velenis E, Kriengsiri P, Tsiotras P. Designing a lowcost spacecraft simulator. IEEE Control Systems Magazine 2003; 23(4):26-37.

5. Lee AY, Wertz JA. In-flight estimation of the Cassini spacecraft's inertia tensor. Journal of Spacecraft and Rockets 2002; 39(1):153-155.
6. Peck MA. Mass-properties estimation for spacecraft with powerful damping. Advances in the Astronautical Sciences 2000; 103(3):2005-2024.

7. Tanygin S, Williams T. Mass property estimation using coasting maneuvers. Journal of Guidance, Control, and Dynamics 1997; 20(4):625-632.

8. van Huffel S, Vandewalle J. The Total Least Squares Problem: Computational Aspects and Analysis. SIAM: Philadelphia, PA, 1991.

9. Soderstrom T, Soverini U, Mahata K. Perspectives on errorsin-variables estimation for dynamic systems. Signal Processing 2002; 82:1139-1154.

10. van Huffel S, Lemmerling P. Total Least Squares Techniques and Errors-in-Variables Modeling: Analysis, Algorithms and Applications. Kluwer Academic Publisher: Dordrecht, 2002.

11. Jun BE, Bernstein DS. Least-correlation estimates for errorsin-variables models. International Journal of Adaptive Control and Signal Processing 2006; 20(4):337-351.

12. Jun BE, Bernstein DS. Extended least-correlation estimates for errors-in-variables nonlinear models. International Journal of Control 2007; 80(2)256-267.

13. Bergmann EV, Walker BK, Levy DR. Mass property estimation for control of asymmetrical satellites. Journal of Guidance, Control, and Dynamics 1987; 10(5):483-491.

14. Schwartz JL, Peck MA, Hall CD. Historical review of airbearing spacecraft simulators. Journal of Guidance, Control, and Dynamics 2003; 26(4):513-522.

15. Ljung L. System Identification-Theory for the User (2nd edn). Prentice-Hall: Englewood Cliffs, NJ, 1999.

16. Ahmed J, Coppola VT, Bernstein DS. Adaptive asymptotic tracking of spacecraft attitude motion with inertia matrix identification. Journal of Guidance, Control, and Dynamics 1998; 21(5):684-691.

17. Johansson R. System Modeling and Identification. PrenticeHall: Englewood Cliffs, NJ, 1993.

18. Koh T, Powers EJ. Second-order Volterra filtering and its application to nonlinear system identification. IEEE Transactions on Acoustics, Speech, and Signal Processing 1985; ASSP-33(6):1445-1455. 